\title{
Journal of Personalized Medicine
}

an Open Access Journal by MDPI
IMPACT
FACTOR
CITESCORE
3.508
1.8 


\section{Journal of}

Personalized

Medicine

an Open Access Journal by MDPI

Editors-in-Chief

Prof. Dr. Stephen B. Liggett

Prof. Dr. David Alan Rizzieri

\begin{abstract}
Message from the Editor-in-Chief
Journal of Personalized Medicine is one of the few journals that covers the diverse areas involved in the field, including research at basic, translational, and clinical levels. Itfocuses on "omics"-level studies that seek to define the basis of interindividual variation in susceptibility for a disease, its prognosis or definition of clinical subsets, and response to therapy (pharmacogenomics). We are also interested in systems biology as it relates to interindividual variation, and research on new methodologies, informatics, and biostatistics, in the aforementioned areas.
\end{abstract}

\section{Author Benefits}

Open Access Unlimited and free access for readers

C No Copyright Constraints Retain copyright of your work and free use of your article

\section{\& Thorough Peer-Review}

$\sqsubseteq$ No Space Constraints, No Extra Space or Color Charges No restriction on the length of the papers, number of figures or colors

$\square[$ Coverage by Leading Indexing Services Scopus, SCIE (Web of Science), PubMed, PMC, Embase, and other databases

Rapid Publication First decision provided to authors approximately 20.1 days after submission; acceptance to publication is undertaken in 2.8 days (median values for papers published in this journal in the first half of 2022) 


\section{Aims and Scope}

For a comprehensive perspective of primary research, commentaries, and reviews related to personalized medicine, also termed precision medicine, JPM aims to integrate expertise from the molecular and translational sciences, therapeutics and diagnostics, as well as discussions of regulatory, social, ethical and policy aspects.

Topics of interest with a view towards personalized medicine: Omics-based studies of disease risk, disease prognosis, and response to therapy

Biomarker identification and application

Drug target discovery and integration with individualized therapy

Pharmacogenomics - genetics and biochemistry of drug uptake, action, and metabolism

Prediction-based drug safety

Novel therapeutics: genetic-based, nanotechnology, targeting strategies

Advancements in Technologies and Resources (genetic testing, expression analysis, microbiomes, genomewide association studies, bioinformatics and health informatics, biostatistics)

Personalization of omics-based non-drug related health interventions

Regulation and bioethics in personalized medicine

\section{Editorial Office}

JPM Editorial Office jpm@mdpi.com MDPI, St. Alban-Anlage 66 4052 Basel, Switzerland Tel: +41616837734 www.mdpi.com mdpi.com/journal/jpm 


\section{GSPA}

The Association of Learned \& Profession Society Publishers

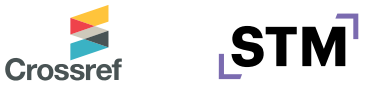

Society for Scholarly Publishing
$|\mathbf{C}| \mathbf{O}|\mathbf{P}| \mathbf{E}$

SPARC*

Europe

\section{DOAJ}

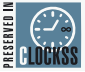

ORCID

\section{Follow}

f facebook.com/MDPIOpenAccessPublishing

twitter.com/MDPIOpenAccess

in linkedin.com/company/mdpi

(O) instagram.com/mdpiopenaccess

$\boldsymbol{6}^{\text {") }}$ weibo.com/mdpicn

Wechat:MDPI-China

\section{Subscribe}

blog.mdpi.com 